\title{
Ultrabroadband background-free coherent anti-Stokes Raman scattering microscopy based on a compact Er:fiber laser system
}

\author{
Romedi Selm, ${ }^{1}$ Martin Winterhalder, ${ }^{1}$ Andreas Zumbusch,${ }^{1, *}$ Günther Krauss, ${ }^{2}$ Tobias Hanke, ${ }^{2}$ \\ Alexander Sell, ${ }^{2}$ and Alfred Leitenstorfer ${ }^{2}$ \\ ${ }^{1}$ University of Konstanz, Department of Chemistry, D-78457 Konstanz, Germany \\ ${ }^{2}$ University of Konstanz, Department of Physics, D-78457 Konstanz, Germany \\ *Corresponding author: andreas.zumbusch@uni-konstanz.de
}

Received May 18, 2010; revised August 3, 2010; accepted August 13, 2010; posted September 3, 2010 (Doc. ID 128612); published September 28, 2010

\begin{abstract}
We demonstrate a scheme for efficient coherent anti-Stokes Raman scattering (CARS) microscopy free of nonresonant background. Our method is based on a compact Er:fiber laser source. Impulsive excitation of molecular resonances is achieved by an $11 \mathrm{fs}$ pulse at $1210 \mathrm{~nm}$. Broadband excitation gives access to molecular resonances from $0 \mathrm{~cm}^{-1}$ up to $4000 \mathrm{~cm}^{-1}$. Time-delayed narrowband probing at $775 \mathrm{~nm}$ enables sensitive and high-speed spectral detection of the CARS signal free of nonresonant background with a resolution of $10 \mathrm{~cm}^{-1}$. (c) 2010 Optical Society of America

OCIS codes: $\quad 300.6230,190.7110,140.3510,140.3500,170.5810$.
\end{abstract}

Coherent anti-Stokes Raman scattering (CARS) is a new type of vibrational microscopy. As such, it is a label-free technique that has lately attracted much attention, mainly for biological applications [1-ㅗㄱ. Two obstacles prevent a broader use of CARS microscopy so far, namely, the presence of a nonresonant electronic background and the complexity of the excitation sources. Several schemes for background removal have been demonstrated, all of which, however, have limitations in practice. With respect to excitation sources, recent advances in fiber laser technology have led to smaller CARS microscopes with improved stability and reliability [ㄴ,5].

In the CARS process, molecules are excited by two input light fields, $\omega_{\text {pump }}$ and $\omega_{\mathrm{S}}$, whose frequency difference is chosen to match the frequency of Raman active transitions. These pulses lead to a coherent vibrational excitation. A probe field $\omega_{\text {probe }}$ is scattered off the molecules to produce the blueshifted anti-Stokes signal at $\omega_{\mathrm{AS}}$. The third-order nonlinearity of the process necessitates the use of pulsed laser sources, which are collinearly focused tightly onto the sample [].

The molecular specificity of the CARS signal is often reduced by a large nonresonant background (NRB). The NRB originates from the third-order nonlinear susceptibility $\chi^{(3)}$ of the specimen. Different experimental approaches have been proven to suppress the NRB, including polarization control [7], epidetection [8], heterodyne detection [9-11], phase contrast [12], and timeresolved CARS $[\overline{13}, \overline{14}]$. These methods either strongly attenuate the resonant CARS signal or are difficult to implement for investigations of biological samples.

In this Letter, we present a CARS microscope that exploits the high flexibility and stability of ultrafast Er:fiber technology to provide optimum pulse shapes in the time and frequency domains for efficient NRB suppression. The setup has a bandwidth of $4000 \mathrm{~cm}^{-1}$ and covers the entire region of molecular vibrational spectra in a single laser pulse.

Our idea is based on time-resolved CARS microscopy and is sketched in Fig. 1. Here, the fact is exploited that

Konstanzer Online-Publikations-System (KOPS) the purely electronic NRB decays instantaneously after the excitation fields are turned off. The vibrational coherences, by contrast, typically persist on a picosecond time scale [15]. Thus, delaying the probe pulse from the excitation results in efficient background suppression. Pulses with an approximate Gaussian shape were employed previously for excitation and probing. Efficient background reduction then also results in attenuation of the resonant signal because the rise times of Gaussian picosecond pulses, which are necessary for obtaining good spectral resolution, are also in the picosecond range. In the scheme proposed here, an ultrashort laser pulse of a duration of 11 fs in the focus excites the vibrational modes of the sample molecules impulsively. Subsequently, a rectangular flattop pulse with a duration of $3 \mathrm{ps}$ is used as a probe pulse (see Fig. 1). The very short rise time of $200 \mathrm{fs}$ of the flattop pulse now allows us to place the probe pulse immediately after excitation. Thus, this approach suppresses the background efficiently, while nearly all of the resonant signal is recovered. We note that a similar excitation scheme has been shown for spectroscopic applications of CARS using pulse shaping techniques [16]. By contrast, our approach is based exclusively on passive optics and is much easier to implement.

In our experimental setup, a mode-locked Er:fiber laser oscillator at a repetition rate of $40 \mathrm{MHz}$ seeds two
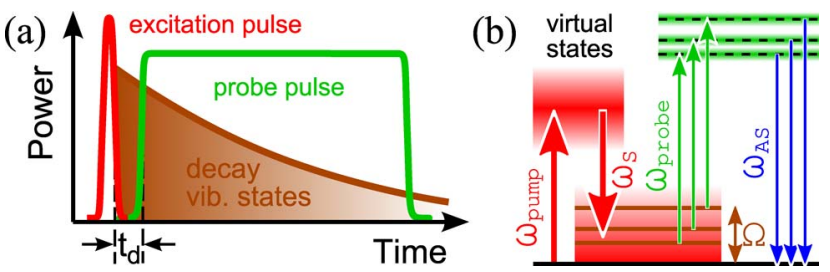

Fig. 1. (Color online) (a) Time-resolved excitation scheme. (b) Excitation scheme in the frequency domain. Impulsive excitation of the vibrational modes by an ultrashort broadband excitation pulse containing $\omega_{\text {pump }}$ and $\omega_{\mathrm{S}}$. A time-delayed narrowband flattop pulse $\omega_{\text {probe }}$ probes the vibrational states oscillating at $\Omega$. The CARS signal $\omega_{\mathrm{AS}}$ carries the spectral information of the Raman active resonances. 
parallel Er:fiber amplifiers. The average output power amounts to $350 \mathrm{~mW}$, corresponding to $8.8 \mathrm{~nJ}$ of pulse energy for each branch. The pulse duration is $130 \mathrm{fs}$ after compression by a Si-prism sequence in free space.

As the two branches share the same seed source, they are synchronized with attosecond precision [17]. The first laser branch is designed to deliver the broadband pulse for the impulsive excitation in the CARS process. It contains the pump $\omega_{\text {pump }}$ and Stokes $\omega_{\mathrm{S}}$ frequency components. The amplifier output is coupled into an assembly of a standard telecom fiber spliced to a highly nonlinear fiber. The fundamental input at $1550 \mathrm{~nm}$ is split into two components. The dispersive wave shifts toward the visible, and the solitonic wave tunes toward longer wavelengths [18]. Adjustment of the input chirp of the driving pulses by translating one prism in the Si compressor allows us to set the center wavelength of the two components [19]. The bandwidth of the dispersive wave amounts to $500 \mathrm{~nm}$ at a center wavelength of $1210 \mathrm{~nm}$, corresponding to $4000 \mathrm{~cm}^{-1}$. The average power amounts to $13 \mathrm{~mW}$. A pulse duration of $7.8 \mathrm{fs}$ has been shown with this arrangement using all-reflective focusing optics [18]. To achieve submicrometer spatial resolution, we employ a water immersion objective ( $32 \times$, NA 0.85$)$. Additional dispersion compensation yields a pulse duration of $(11.4 \pm 1.0)$ fs in the focus. Pulse measurements are taken by a collinear second-harmonic-generation frequency-resolved optical gating with subsequent phase retrieval (see Fig. 2).

The second branch delivers the probe pulse in the CARS excitation scheme. The fundamental output of the amplifier is focused in a periodically poled lithium niobate
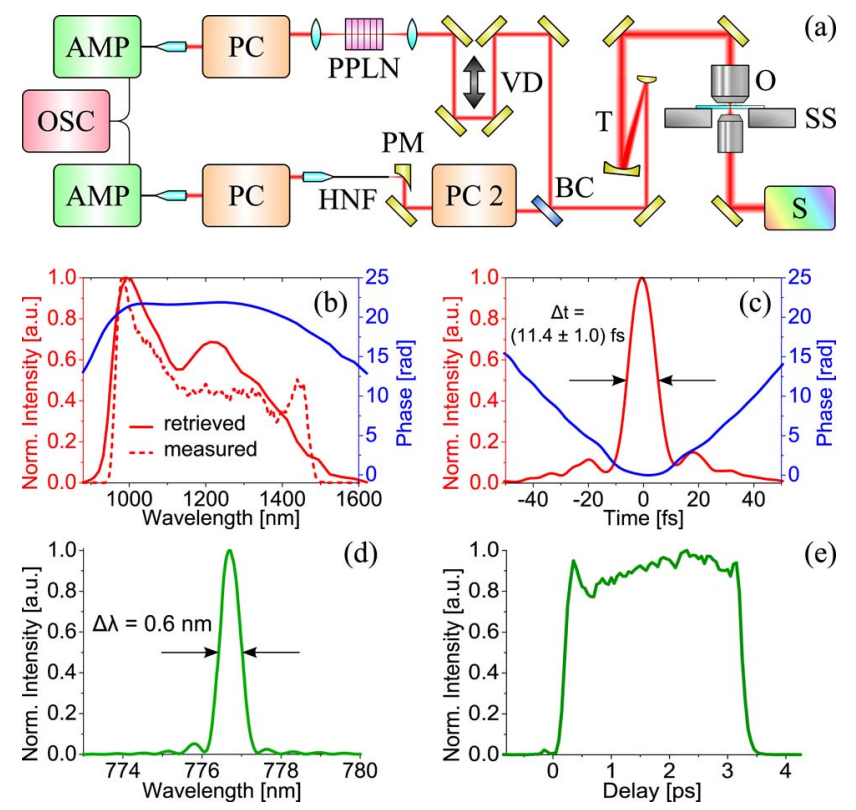

Fig. 2. (Color online) (a) Schematic setup: OSC, fs-Er:fiber oscillator; AMP, erbium-doped fiber amplifier; PC, silicon prism compressor; PC2, SF10 prism compressor; HNF, highly nonlinear germanosilicate fiber for frequency conversion; PM, parabolic mirror; PPLN, periodically poled lithium niobate crystal; VD, variable delay line; $\mathrm{BC}$, dichroic beam combiner; $\mathrm{T}$, telescope; O, objective; SS, scanning stage; S, spectrometer. (b) and (c) Spectrum and frequency-resolved optical gating data of excitation pulse. (d) Spectrum of probe pulse. (e) Probe pulse shape in time measured by a cross-correlation in the focus.
(PPLN) crystal for second-harmonic generation. A crystal length of $10 \mathrm{~mm}$ is used to generate flattop pulses of $3 \mathrm{ps}$ duration, $200 \mathrm{fs}$ rise time, and a bandwidth of $0.6 \mathrm{~nm}$ close to the bandwidth limit [Figs. 2(d) and 2(e)]. The bandwidth corresponds to $10 \mathrm{~cm}^{-1}$, which matches the typical linewidths of vibrational bands in the condensed phase [8]. Average powers of $50 \mathrm{~mW}$ are obtained at $776.7 \mathrm{~nm}$. The two laser beams are spatially combined by a dichroic mirror and coupled into a home-built scanning stage microscope. Temporal control is implemented by a variable delay line. The broadband CARS signal is collected by a glass objective (Leica, 20×, NA 0.7), passed through a spectrometer, and detected by an avalanche photodiode or a CCD camera.

To demonstrate the principle behind the time-resolved excitation scheme, a liquid film of benzonitrile is studied. A block of time-resolved spectra is shown in Fig. 3. The vertical axis corresponds to the time delay of the leading edge of the probe pulse with respect to the excitation pulse. Before time zero down to -3 ps, the excitation and probe pulses are simultaneously passing the sample. In this region, the CARS signal is obstructed by a broad NRB signal, rendering spectral interpretation difficult. After time zero, the dispersive lineshape due to the NRB disappears instantaneously, while the retarded vibrational bands persist. The emission becomes much more distinct and resembles a spontaneous Raman spectrum. Relative intensities of the bands are influenced by the vibrational coherence times of the different modes. Determination of lifetimes of different vibrational states is possible by analyzing the time-resolved spectra. The large bandwidth of our excitation pulse allows us to reach a spectral window corresponding to the entire range of vibrational frequencies (Fig. 3). The roll-off of the phase above $1300 \mathrm{~nm}$, however, makes it necessary to include residual pump light at $1550 \mathrm{~nm}$ to address the $\mathrm{CH}$ stretching modes. The short rise time of the probe pulse enables us to efficiently pick up these resonances, which have very short lifetimes. A spectral resolution of $10 \mathrm{~cm}^{-1}$ is provided by the narrowband probe.

The new excitation scheme is readily employed in imaging applications, as demonstrated in Fig. 4 on C. elegans in water. Background suppression is achieved by delaying the probe pulse with respect to the excitation pulse. The lipid distribution becomes visible by tuning the monochromator to the aliphatic $\mathrm{CH}$ resonance at

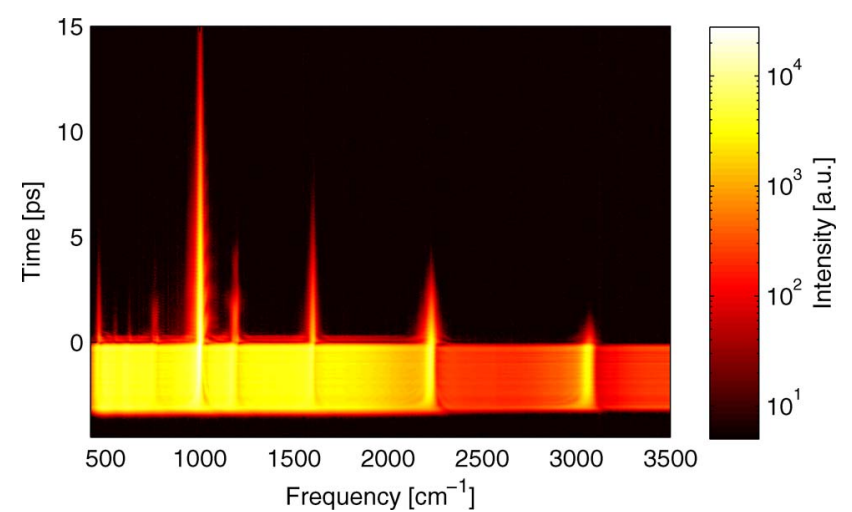

Fig. 3. (Color online) Time-resolved spectrum of Benzonitrile. Acquisition time $0.54 \mathrm{~ms} /$ spectrum, $0.97 \mathrm{~s}$ for all 1800 spectra with CCD recording. 

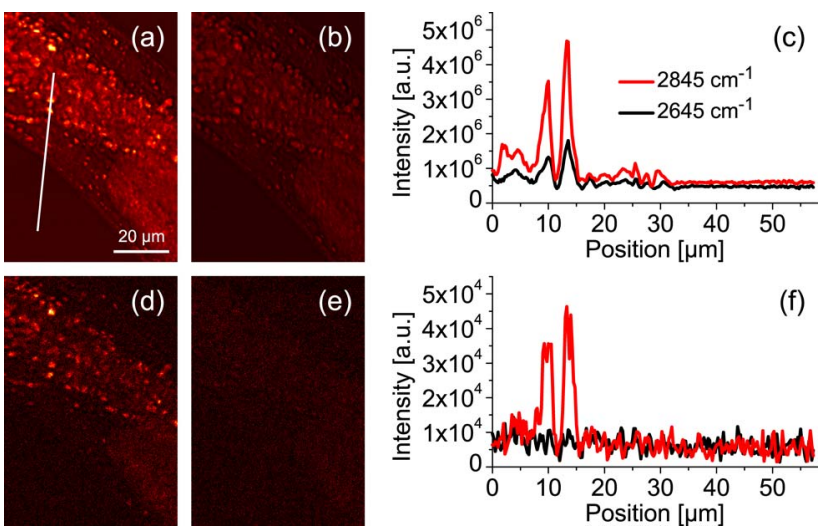

Fig. 4. (Color online) Imaging of the lipid distribution of C. elegans. (a) Image recorded at the $\mathrm{CH}$ resonance at $2845 \mathrm{~cm}^{-1}$ with NRB. (b) Image recorded off resonance at $2645 \mathrm{~cm}^{-1}$ with NRB. (c) Profile plot with NRB along the line drawn in (a). (d) Image recorded at $2845 \mathrm{~cm}^{-1}$ with NRB suppression. (e) Image recorded off resonance at $2645 \mathrm{~cm}^{-1}$ with NRB suppression. (f) Profile plot with NRB suppression along the line drawn in (a).

$2845 \mathrm{~cm}^{-1}$ [Fig. 4(d)]. When the detection window of the monochromator is detuned from resonance, the contrast disappears [Fig. 4(e)]. This shows that our CARS detection scheme strongly suppresses the NRB. Note that imaging of bands with lifetimes below 300 fs leads to decreasing signal intensities. For comparison, images recorded without NRB suppression, i.e., with temporal overlap of excitation and probe pulse, are depicted in Figs. 4(a) and 4(b). Working on resonance, the lipid distribution is still discernible [Fig. 4(a)], but the contrast is reduced as compared with Fig. 4(d). Unlike Fig. 4(e), some background also remains under nonresonant probing [Fig. 4(b)].

In conclusion, we present a scheme for highly efficient CARS microscopy free of nonresonant background. Our technique is based on a time-resolved CARS experiment. An Er:fiber laser is used as a compact and rugged excitation source, offering turn-key operation. The broadband excitation pulse reaches vibrational levels from 0 up to $4000 \mathrm{~cm}^{-1}$, while narrowband probing maintains a high spectral resolution of $10 \mathrm{~cm}^{-1}$. The short rise time of only 200 fs of our probe pulse enables nonresonant background-free recording of CARS spectra with minimal loss of resonant signal. Furthermore, precise determination of vibrational lifetimes even for short-lived resonances is possible. Passive dispersion management allows the use of high-NA transmissive objectives.

Financial support from the Baden-Württemberg Stiftung is gratefully acknowledged.

\section{References}

1. A. Volkmer, J. Phys. D 38, R59 (2005).

2. C. Evans and X. S. Xie, Annu. Rev. Anal. Chem. 1, 883 (2008).

3. M. Müller and A. Zumbusch, Chem. Phys. Chem. 8, 2156 (2007).

4. M. Marangoni, A. Gambetta, C. Manzoni, V. Kumar, R. Ramponi, and G. Cerullo, Opt. Lett. 34, 3262 (2009).

5. G. Krauss, T. Hanke, A. Sell, D. Träutlein, A. Leitenstorfer, R. Selm, M. Winterhalder, and A. Zumbusch, Opt. Lett. 34, 2847 (2009).

6. A. Zumbusch, G. R. Holtom, and X. S. Xie, Phys. Rev. Lett. 82, 4142 (1999).

7. J.-X. Cheng, L. D. Book, and X. S. Xie, Opt. Lett. 26, 1341 (2001).

8. J. X. Cheng, A. Volkmer, L. D. Book, and X. S. Xie, J. Phys. Chem. B 105, 1277 (2001).

9. T. W. Kee, H. X. Zhao, and M. T. Cicerone, Opt. Express 14, 3631 (2006).

10. E. O. Potma, C. L. Evans, and X. S. Xie, Opt. Lett. 31, 241 (2006).

11. M. Jurna, J. P. Korterik, C. Otto, J. L. Herek, and H. L. Offerhaus, Opt. Express 16, 15863 (2008).

12. M. Jurna, J. P. Korterik, C. Otto, J. L. Herek, and H. L. Offerhaus, Phys. Rev. Lett. 103, 043905 (2009).

13. A. Volkmer, L. D. Book, and X. S. Xie, Appl. Phys. Lett. 80, 1505 (2002).

14. Y. J. Lee and M. T. Cicerone, Appl. Phys. Lett. 92, 041108 (2008).

15. A. Laubereau and W. Kaiser, Rev. Mod. Phys. 50, 607 (1978).

16. D. Pestov, R. K. Murawski, G. O. Ariunbold, X. Wang, M. Zhi, A. V. Sokolov, V. A. Sautenkov, Y. V. Rostovtsev, A. Dogariu, Y. Huang, and M. O. Scully, Science 316, 265 (2007).

17. F. Adler, A. Sell, F. Sotier, R. Huber, and A. Leitenstorfer, Opt. Lett. 32, 3504 (2007).

18. A. Sell, G. Krauss, R. Scheu, R. Huber, and A. Leitenstorfer, Opt. Express 17, 1070 (2009).

19. F. Tauser, F. Adler, and A. Leitenstorfer, Opt. Lett. 29, 516 (2004). 